\title{
CLUSTER INFORMATION DISCLOSURE IN STRATEGIC PLANNING OF REGION'S ECONOMY
}

\author{
Aleksandr E. Vorobev \\ Volgograd State University, Volgograd, Russian Federation
}

\begin{abstract}
The article represents the results of the analysis of existing approaches to describing economic clusters. These approaches use self-dependent characteristics that complicate evaluating and comparing clusters. Consequently, the author offers the concept of information disclosure of a cluster and its overall indicator. This indicator enables to evaluate clusters, to define a stage of their functioning and to compare results of their activity with other clusters. The existing Russian methods of describing clusters form the basis for this article. These methods are proposed by the Cluster observatory of Higher School of Economics and the Geographic information system "Industrial Parks, Science and Technology Parks and Clusters" of the Russian Federation. The author singles out three aspects of characteristics: attributive, quantitative, qualitative. The characteristics used for calculating the overall indicator of disclosure are quantitative and qualitative. They are generalized and unified using indicators of the project of the Ministry of Economic Development of the Russian Federation "Development of the Innovation Clusters - Leaders of World-Class Investment Attractiveness". The author offers two approaches to using the information disclosure indicator: dynamic and reference. The dynamic approach enables to define a stage of cluster development using the relation of the current condition to the previous one. The reference approach defines cluster development in relation to a standard that describes its global competitiveness. Both offered approaches can be used by regional and federal government bodies as well as cluster management for taking strategic decisions on the region development.

Key words: cluster, regional economy, strategic cluster policy, strategic planning, information disclosure, regional development, instrument of strategic development, characteristics of clusters.
\end{abstract}

Citation. VorobevA.E. Cluster Information Disclosure in Strategic Planning of Region's Economy. Journal of Volgograd State University. Economics, 2019, vol.21, no. 4, pp. 118-125. (in Russian). DOI: https://doi.org/10.15688/ek.jvolsu.2019.4.12

\section{ИНФОРМАЦИОННОЕ ОТРАЖЕНИЕ КЛАСТЕРА В СТРАТЕГИЧЕСКОМ ПЛАНИРОВАНИИ ЭКОНОМИКИ РЕГИОНА}

\author{
Александр Евгеньевич Воробьев \\ Волгоградский государственный университет, г. Волгоград, Российская Федерация
}

\begin{abstract}
Аннотация. В статье представлены результаты анализа существующих подходов к описанию экономических кластеров. Их недостаток состоит в том, что используются отдельные характеристики, что затрудняет анализ динамики кластера, сравнение кластеров друг с другом, принятие решений относительно развития кластера и региона. Предложено понятие информационного отражения кластера и его обобщающего показателя. Последний позволит оценить кластеры, определить стадию их функционирования и сопоставить результаты их деятельности с другими кластерами в региональном или отраслевом контексте. В основу предлагаемой методики положены существующие отечественные методики описания кластеров, предлагаемые Кластерной обсерваторией Высшей школы экономики и Геоинформационной системой «Индустриальные парки, технопарки, кластеры». Было выделено три группы характеристик кластера: атрибутивные, количественные и качественные. Для расчета обобщающего показателя информационного (2) отражения в работе предлагается использовать качественные и количественные характеристики, которые
\end{abstract}


приведены к единому виду с использованием целевых показателей проекта Минэкономразвития России «Развитие инновационных кластеров - лидеров инвестиционной привлекательности мирового уровня». Предложено два подхода к использованию показателя информационного отражения кластера: динамический и нормативный. Динамический подход позволяет определить, на какой стадии функционирования находится кластер, исходя из отношения его текущего состояния к предыдущему. Нормативный подход позволяет определить уровень развития кластера относительно эталона, что отражает его глобальную конкурентоспособность. Оба предложенных подхода могут быть использованы региональными и федеральными органами исполнительной власти, а также руководящими структурами кластеров для принятия адресных стратегических решений по развитию региона.

Ключевые слова: кластер, региональная экономика, стратегическая кластерная политика, стратегическое планирование, информационное отражение, развитие регионов, инструмент стратегического развития, характеристика кластеров.

Цитирование. Воробьев А. Е. Информационное отражение кластера в стратегическом планировании экономики региона // Вестник Волгоградского государственного университета. Экономика. - 2019. - Т. 21, № 4. - C. 118-125. - DOI: https://doi.org/10.15688/ek.jvolsu.2019.4.12

\section{Постановка проблемы}

На современном этапе совершенствование экономики России направлено на повышение уровня ее конкурентоспособности, расширение импортозамещения, увеличение объемов экспорта и доли добавленной стоимости в нем. Решению указанных задач способствует развитие кластерных форм организации экономической деятельности, создающих новые условия для производства конечных товаров и услуг и их реализации на мировых рынках [Идентификация кластера..., 2015].

В зарубежной и отечественной литературе существует множество подходов к определению понятия «кластер», которые систематизировал Л.С. Марков [Марков, 2015]. В основу данной работы положена дефиниция М. Портера: «Кластер - это группа географически соседствующих взаимосвязанных компаний (поставщики, производители и др.) и связанных с ними организаций (образовательные заведения, органы государственного управления, инфраструктурные компании), действующих в определенной сфере и взаимодополняющих друг друга» [Портер, 2005]. В результате анализа М. Портером конкурентных особенностей различных отраслей было выявлено, что конкурентоспособные международные фирмы базируются в одной стране, зачастую в одном ее регионе. Это объясняется тем, что компании, достигнув конкурентоспособности на глобальном рынке, начинают положительно воздействовать на свое ближайшее окружение. А их успехи и достижения соответственно влияют на рост конкурентоспособности данной фирмы в дальнейшем [Портер, 1993].

Кластерная политика становится важным направлением экономической политики, направленной на формирование благоприятных условий и концентрации ресурсов предпринимательства, проявляясь во всех сферах, родах и видах деятельности в той или иной мере. Данная политика для своего научного обоснования и успешной реализации нуждается в решении теоретических и методических проблем релевантного информационного отражения кластеров как локализованных ассоциативных форм взаимодействия групп фирм в процессе производства конечного товара.

Объективными предпосылками создания и развития кластеров на территории региона являются высокий уровень развития отрасли, в которой функционирует кластер, доступ к природным ресурсам, наличие высококлассных управленцев и специалистов, существование научно-исследовательской базы для развития новых технологий [Миролюбова, 2013]. К субъективным предпосылкам следует отнести государственное стимулирование подобных объединений предприятий, а также стратегические и тактические решения в отношении функционирующих кластеров. Данные решения могут приниматься как органами государственной власти, так и самими кластерами. Обоснованность таких решений будет зависеть от информационного отражения кластера.

Под информационным отражением понимается совокупность актуальных и реле- 
вантных данных по функционированию кластера в любом временном интервале. Так как кластеры являются одним из институтов развития регионов, решения, принимаемые в адрес кластеров, будут иметь для них стратегическое значение.

\section{Методологические основы}

\section{информационного отражения кластера}

В работе предлагается сформировать обобщенный показатель информационного отражения кластера, используемый в оценке его функционирования, а также в проведении сравнительного анализа кластеров, вне зависимости от сферы их деятельности.

В данный момент на практике для описания результатов деятельности кластеров применяются отдельные характеристики. Также существуют несовпадения, а порой и противоречия в информации, предоставляемой о результатах кластеризации отечественной экономики [Иншаков и др., 2017]. Анализ данных о кластерах в России, предоставляемых Российской кластерной обсерваторией Высшей школы экономики [Реестр. Карта кластеров России, 2019] и Геоинформационной системой «Индустриальные парки, технопарки, кластеры» [Реестр промышленных кластеров, 2019], позволил выделить три группы характеристик: атрибутивные, качественные и количественные. Атрибутивные содержат информационную справку о кластере: название, субъект РФ, год создания, сайт, ключевая и сопутствующие специализации, цель объединения кластера, приоритеты развития кластера, краткое описание основной продукции, описание менеджмента кластера, презентационные материалы и документы кластера, проекты кластера, предложения для других кластеров. Качественные характеристики описывают уровень развития кластера, статус кластера, участников и партнеров кластера.

К количественным характеристикам можно отнести: объем совокупной выручки от продаж участниками кластеров на экспорт несырьевой продукции; добавленную стоимость, создаваемую участниками кластера; объем затрат на научные исследования и разработки; выработка на одного работника; количество созданных или модернизированных высокопроизводительных рабочих мест (ВРМ); количество технологических стартапов, которые привлекли инвестиции.

Обобщающим показателем информационного отражения кластера будет являться коэффициент $K$, рассчитываемый по формуле: $K=\sum_{i=1}^{j} K_{i}$, где $K_{i}$ - значение $i$-й характеристики кластера; $j$ - количество характеристик кластера. Данный показатель позволяет оценить текущее состояние кластера.

Десять количественных и качественных характеристик приведены к значениям, находящимся в интервале [0;1] (см. табл. 1), где 1 означает максимальное соответствие данной характеристике, значение 0 - минимальное соответствие данной характеристике, значение 0,5 - среднее соответствие данной характеристике.

Пояснения по возможным значениям $K_{i}$.

Характеристики $K_{1}-K_{3}$ могут принимать только три значения $(0,1 ; 0,5 ; 1)$, характеристики $K_{4}-K_{10}$ могут принимать значения во всем интервале $[0 ; 1]$.

Характеристика уровня развития кластера $K_{1}$ принимает значение 0 - если кластер находится на начальном уровне организационного развития; 0,5 - если на среднем уровне; 1 - если на высоком.

Характеристика статуса кластера $K_{2}$ принимает значение 0 , если кластер не включен в перечень поддерживаемых кластеров Минпромторг России и не поддерживается центром кластерного развития; 0,5 - если кластер поддерживается одной из двух структур; 1 - если включен в перечень поддержки обеих структур.

Характеристика партнеров кластера $K_{3}$ может принимать значения: 0 - если у кластера нет партнеров среди предприятий, не входящих в кластер; 0,5 - если у кластера есть только российские партнеры; 1 - если у кластера есть и российские, и зарубежные партнеры.

Характеристика участников кластера $K_{4}$ принимает значения в интервале $[0 ; 1]$ в зависимости от количества организаций, входящих в кластер. Один участник кластера добавляет 0,01 к значению $K_{4}$. Для кластеров, имеющих в своем составе 100 и более участников, значение характеристики равно 1. 
A.Е. Воробьев. Информационное отражение кластера в стратегическом планировании экономики региона

Таблища 1

Характеристики информационного отражения кластера

\begin{tabular}{|l|l|c|}
\hline \multicolumn{1}{|c|}{ Характеристика } & \multicolumn{1}{|c|}{ Описание } & $\begin{array}{c}\text { Возможные } \\
\text { значения } K_{i}\end{array}$ \\
\hline $\begin{array}{l}\text { Уровень развития кла- } \\
\text { стера }\left(K_{1}\right)\end{array}$ & Уровень организационного развития кластера & $0 ; 0,5 ; 1$ \\
\hline Статус кластера $\left(K_{2}\right)$ & $\begin{array}{l}\text { Сведения о включении кластера в перечень поддерживаемых кла- } \\
\text { стеров Министерством промышленности и торговли РФ, поддерж- } \\
\text { ке центром кластерного развития }\end{array}$ & $0 ; 0,5 ; 1$ \\
\hline Партнеры кластера $\left(K_{3}\right)$ & Российские и зарубежные партнеры кластера & $0 ; 0,5 ; 1$ \\
\hline Участники кластера $\left(K_{4}\right)$ & Количество организаций-участников кластера & {$[0 ; 1]$} \\
\hline $\begin{array}{l}\text { Выручка от продажи то- } \\
\text { варов и услуг }\left(K_{5}\right)\end{array}$ & $\begin{array}{l}\text { Общая выручка от продаж участниками кластера на экспорт не- } \\
\text { сырьевой продукции }\end{array}$ & {$[0 ; 1]$} \\
\hline $\begin{array}{l}\text { Добавленная стоимость } \\
\left(K_{6}\right)\end{array}$ & $\begin{array}{l}\text { Увеличение компаниями кластера доли добавленной стоимости в } \\
\text { выручке }\end{array}$ & {$[0 ; 1]$} \\
\hline $\begin{array}{l}\text { Технологические стар- } \\
\text { тапы }\left(K_{7}\right)\end{array}$ & $\begin{array}{l}\text { Количество технологических стартапов, которые привлекли инве- } \\
\text { стиции }\end{array}$ & {$[0 ; 1]$} \\
\hline $\begin{array}{l}\text { Выработка на одного } \\
\text { работника }\left(K_{8}\right)\end{array}$ & $\begin{array}{l}\text { Отношение количества произведенной продукции и количеству ра- } \\
\text { ботников }\end{array}$ & {$[0 ; 1]$} \\
\hline Количество ВРМ $\left(K_{9}\right)$ & Количество ВРМ, созданных заново или в результате модернизации & {$[0 ; 1]$} \\
\hline $\begin{array}{l}\text { Научные исследования и } \\
\text { разработки }\left(K_{10}\right)\end{array}$ & $\begin{array}{l}\text { Объем работ в области научных исследований и разработок, вы- } \\
\text { полняемых двумя и более организациями совместно }\end{array}$ & {$[0 ; 1]$} \\
\hline
\end{tabular}

Примечание. Составлено по: [Реестр. Карта кластеров России..., 2019], [Реестр промышленных кластеров..., 2019].

Показатели характеристик $K_{5}-K_{10}$, принимающие значения в интервале [0;1], предлагается вычислять в зависимости от степени выполнения целевых показателей проекта Минэкономразвития России «Развитие инновационных кластеров - лидеров инвестиционной привлекательности мирового уровня» [Инновационные кластеры..., 2016] (табл. 2).

Расчет коэффициента $K$ возможен не только для кластеров, входящих в данный при- оритетный проект, так как для его расчета используются универсальные характеристики. Итоговый коэффициент К может принимать значения в интервале [0;10].

Для полноты информационного отражения кластеров, а также для осуществления мониторинга эффективности мероприятий по развитию кластера необходимо получать и обрабатывать не только данные о текущем состоянии кластера, но и информацию о тенденциях его развития.

Таблица 2

Характеристики информационного отражения кластера, зависящие от степени выполнения целевых показателей проекта МЭР

\begin{tabular}{|c|c|c|}
\hline Характеристика & $\begin{array}{c}\text { Целевой показатель } \\
\text { проекта }\end{array}$ & Возможные значения $K_{i}$ \\
\hline $\begin{array}{l}\text { Выручка от продажи това- } \\
\text { ров и услуг }\end{array}$ & Увеличение в 2 раза & $\begin{array}{l}\text { Значение } K_{i} \text { увеличивается на } 0,1 \text { с увеличением вы- } \\
\text { ручки на } 10 \%\end{array}$ \\
\hline Добавленная стоимость & Увеличение на 20 \% & $\begin{array}{l}\text { Значение } K_{i} \text { увеличивается на } 0,1 \text { с увеличением добав- } \\
\text { ленной стоимости на } 2 \%\end{array}$ \\
\hline Технологические стартапы & 300 единиц & $\begin{array}{l}\text { Значение } K_{i} \text { увеличивается на } 0,1 \text { с увеличением коли- } \\
\text { чества технологических стартапов на } 30 \text { единиц }\end{array}$ \\
\hline $\begin{array}{l}\text { Выработка на одного ра- } \\
\text { ботника }\end{array}$ & Увеличение на $20 \%$ & $\begin{array}{l}\text { Значение } K_{i} \text { увеличивается на } 0,1 \text { с увеличением выра- } \\
\text { ботки на одного работника на } 2 \%\end{array}$ \\
\hline $\begin{array}{l}\text { Высокопроизводительные } \\
\text { рабочие места (ВРМ) }\end{array}$ & 100000 единиц & $\begin{array}{l}\text { Значение } K_{i} \text { увеличивается на } 0,1 \text { с увеличением ВРМ } \\
\text { на } 10000 \text { единиц }\end{array}$ \\
\hline $\begin{array}{l}\text { Научные исследования и } \\
\text { разработки }\end{array}$ & 100 млрд руб. & $\begin{array}{l}\text { Значение } K_{i} \text { увеличивается на } 0,1 \text { с увеличением затрат } \\
\text { на научные исследования и разработки на } 10 \text { млрд руб. }\end{array}$ \\
\hline
\end{tabular}

Примечание. Составлено автором с использованием показателей из [Инновационные кластеры..., 2016]. 


\section{Информационное отражение кластера: авторская методика}

Существует множество состояний кластера $K=\left\{K_{1}, K_{2}, K_{3}, \ldots, K_{n}\right\}$, где $n>3$ - количество рассматриваемых состояний кластера. Изменение состояния кластера обозначается как $Q=K_{j+1} / K_{j}$, где $j-$ номер состояния. Тогда множество изменений состояния кластера можно формализовать следующим образом: $Q=\left\{Q_{1}, Q_{2}, \ldots, Q_{m}\right\}$, где $m=n-1$. Показатель $Q$ является динамическим, так как позволяет оценить кластер с точки зрения: определения стадии развития кластера, на которой он находится («динамический» подход); определения развития кластера относительно «нормы» («нормативный» подход). После данной оценки предлагается комплекс мер для совершенствования кластера. Рассмотрим оба подхода.

1. Динамический подход - отношение состояния кластера к своему предыдущему состоянию, формализованный вид которого: $Q=K_{j+1} / K_{j}$.

Исходя из максимального и минимального значений итогового коэффициента $K, Q$ может принимать значения больше нуля.
Математическое обоснование данного подхода: с учетом значения коэффициента $Q$ можно определить стадию развития кластера.

В исследованиях выделяют шесть стадий развития кластеров: Первичная стадия, Эмбриональный кластер, Развивающийся кластер, Зрелый кластер, Угасающий кластер, Трансформация кластера [Clark, 2013].

Для первичной стадии кластера сравнение с предыдущим состоянием является неактуальным, в то же время возникает вопрос об идентификации состояния кластера, при котором он близок к своей ликвидации, поэтому предлагается дополнить данную модель стадией ликвидации кластера.

Трактовка возможных значений коэффициента $Q$ представлена в таблице 3 .

Выделение ключевых ранних и поздних стадий развития кластера чрезвычайно важно для принятия стратегических решений в адрес региона и самого кластера. Наиболее правильный вариант развития кластера, по мнению исследователей, заключается в постоянном циклическом возврате на стадию развивающегося кластера, что отражает способность кластера изменяться в соответствии с требованиями рыночной экономики.

Таблица 3

Трактовка значений $Q$ для динамического подхода

\begin{tabular}{|c|c|c|}
\hline Значение & Описание & $\begin{array}{c}\text { Стадия развития } \\
\text { кластера } \\
\end{array}$ \\
\hline$Q<0,5$ & $\begin{array}{l}\text { Если показатели кластера уменьшились за рассматриваемый период } \\
\text { более, чем на } 50 \text { \%, то такой кластер находится на стадии ликвида- } \\
\text { ции, нужно принимать срочные меры по восстановлению }\end{array}$ & $\begin{array}{l}\text { Стадия ликвидации } \\
\text { кластера }\end{array}$ \\
\hline $0,5<Q<0,9$ & $\begin{array}{l}\text { Если показатели кластера находятся на уровне } 50-90 \% \text {, кластер пе- } \\
\text { реживает стадию трансформации с последующим ожиданием пере- } \\
\text { хода в одну из стадий развития }\end{array}$ & $\begin{array}{l}\text { Стадия трансфор- } \\
\text { мации кластера }\end{array}$ \\
\hline $0,9<Q<1$ & $\begin{array}{l}\text { Показатели кластера на уровне } 90-100 \text { \% означают, что он находится } \\
\text { на стадии угасающего кластера. Если это цикличный процесс, то это } \\
\text { нормальное явление и в дальнейшем вероятен переход к стадии } \\
\text { трансформации кластера }\end{array}$ & $\begin{array}{l}\text { Стадия угасающе- } \\
\text { го кластера }\end{array}$ \\
\hline $1<Q<1,25$ & $\begin{array}{l}\text { Если значение } ? K \text { превысило } 1 \text { и колеблется в пределах до } 1,25 \text {, то } \\
\text { кластер находится в зрелом состоянии и развивается. Сильные пози- } \\
\text { ции в сфере научных разработок и внедрения инновационных техно- } \\
\text { логий позволяют кластеру переходить из стадии зрелости на стадию } \\
\text { развития, используя научные достижения в качестве драйвера роста }\end{array}$ & $\begin{array}{l}\text { Стадия зрелого } \\
\text { кластера }\end{array}$ \\
\hline $1,25<Q<1,75$ & $\begin{array}{l}\text { Если показатели кластера растут на 25-75 \% от предыдущего перио- } \\
\text { да, значит он находится в стадии развивающегося кластера и при- } \\
\text { ближается к переходу в стадию зрелости }\end{array}$ & $\begin{array}{l}\text { Стадия развиваю- } \\
\text { щегося кластера }\end{array}$ \\
\hline$Q>1,75$ & $\begin{array}{l}\text { Если значение изменения характеристик кластера превышает } 75 \text { \%, } \\
\text { значит кластер только создан либо преодолел стадию трансформа- } \\
\text { ции и выходит на привычные для себя мощности }\end{array}$ & $\begin{array}{l}\text { Стадия эмбрио- } \\
\text { нального кластера }\end{array}$ \\
\hline
\end{tabular}

Примечание. Составлено автором. 
A.E. Воробьев. Информационное отражение кластера в стратегическом планировании экономики региона

2. Нормативный подход - отношение развития кластера к норме (эталону).

В случаях, когда стадия развития для исследуемого кластера определена, существует необходимость определить его позиции в контексте глобальной конкурентоспособности. Для этого предлагается подход, позволяющий сравнить показатель информационного отражения кластера с нормативными (эталонными) значениями. Значение эталона задается как максимально возможное для каждого $K_{i}$ (табл. 4).

Формализованный вид данного подхода: $Q_{\text {эт }}=K_{j} K_{\text {эт }}$, где $K_{j}$ - текущее состояние клас- тера, $K_{\text {эт }}$-эталонное состояние кластера на этой же стадии развития. Так как $K_{\dot{j}}$ принимает значения в пределах интервала $[0,4 ; 10]$, значит множество значений $Q_{\text {эт }}$ лежит в интервале $[0,04 ; 1]$.

Рассмотрим возможные значения $Q_{\text {эт }}$ для данного подхода (табл. 5).

Данный подход также представляет важность для региональных и федеральных политиков, так как позволяет оценить позиции региональных кластерных объединений по сравнению с эталоном, определить слабые места и принимать решения, направленные на их устранение.

Таблица 4

Значение характеристик для эталонного кластера

\begin{tabular}{|l|l|c|}
\hline \multicolumn{1}{|c|}{ Характеристика } & \multicolumn{1}{|c|}{ Описание } & Значение \\
\hline $\begin{array}{l}\text { Уровень развития кла- } \\
\text { стера }\left(K_{1}\right)\end{array}$ & Высокий уровень развития кластера & $K_{1}=1$ \\
\hline Статус кластера $\left(K_{2}\right)$ & $\begin{array}{l}\text { Кластер включен в перечень поддерживаемых кластеров Мини- } \\
\text { стерства промышленности и торговли РФ и поддерживается } \\
\text { центром кластерного развития }\end{array}$ & $K_{2}=1$ \\
\hline Участники кластера $\left(K_{3}\right)$ & В состав кластера входит более 100 организаций & $K_{3}=1$ \\
\hline Партнеры кластера $\left(K_{4}\right)$ & У кластера есть российские и зарубежные партнеры & $K_{4}=1$ \\
\hline $\begin{array}{l}\text { Выручка от продажи то- } \\
\text { варов и услуг }\left(K_{5}\right)\end{array}$ & $\begin{array}{l}\text { Выручка от продажи кластером товаров и услуг увеличилась как } \\
\text { минимум в 2 раза }\end{array}$ & $K_{5}=1$ \\
\hline $\begin{array}{l}\text { Добавленная стоимость } \\
\left(K_{6}\right)\end{array}$ & $\begin{array}{l}\text { Добавленная стоимость, созданная предприятиями-участниками } \\
\text { кластера, увеличилась как минимум на 20\% }\end{array}$ & $K_{6}=1$ \\
\hline $\begin{array}{l}\text { Технологические стар- } \\
\text { тапы }\left(K_{7}\right)\end{array}$ & $\begin{array}{l}\text { Количество технологических стартапов в предприятиях- } \\
\text { участниках кластера составляет не менее 300 единиц }\end{array}$ & $K_{7}=1$ \\
\hline $\begin{array}{l}\text { Выработка на одного } \\
\text { работника }\left(K_{8}\right)\end{array}$ & $\begin{array}{l}\text { Выработка на одного работника на предприятиях-участниках } \\
\text { кластера выросла не менее, чем на 20 \% }\end{array}$ & $K_{8}=1$ \\
\hline Количество ВРМ $\left(K_{9}\right)$ & $\begin{array}{l}\text { Количество высокопроизводительных рабочих мест на предпри- } \\
\text { ятиях-участниках кластера составляет не менее } 100 \text { 000 единиц }\end{array}$ & $K_{9}=1$ \\
\hline $\begin{array}{l}\text { Научные исследования и } \\
\text { разработки }\left(K_{10}\right)\end{array}$ & $\begin{array}{l}\text { Затраты на научные исследования и разработки составляют не } \\
\text { менее 100 млрд рублей }\end{array}$ & $K_{10}=1$ \\
\hline
\end{tabular}

Примечание. Составлено автором.

Таблица 5

Трактовка значений для нормативного подхода

\begin{tabular}{|c|c|}
\hline Значение & Описание \\
\hline$Q_{\text {эт }}<0,3$ & Неудовлетворительное развитие кластера, следует принимать срочные меры \\
\hline $0,3 \leq Q_{\text {эт }}<0,6$ & $\begin{array}{l}\text { Удовлетворительное развитие кластера, однако долгое нахождение на таких позициях гро- } \\
\text { зит кластеру последующим упадком. Рекомендуется проведение мероприятий, направлен- } \\
\text { ных на развитие кластера: увеличение затрат на науку и разработки, привлечение дополни- } \\
\text { тельных инвестиций, работа над расширением экосистемы кластера }\end{array}$ \\
\hline $0,6 \leq Q_{\text {эт }}<0,75$ & $\begin{array}{l}\text { Значение показателя на 25-40 \% ниже эталонного можно считать нормальным, отражаю- } \\
\text { щим планомерное развитие кластера и процессов кластеризации }\end{array}$ \\
\hline $0,75 \leq Q_{\text {эт }}<0,9$ & $\begin{array}{l}\text { Если кластер показывает результаты } 75-90 \text { \% развития от эталона, то можно говорить о } \\
\text { том, что кластер развивается успешно, управление кластером реализуется правильно: име- } \\
\text { ется эффективное производство и устойчивые рынки сбыта, высокий уровень экспорта, } \\
\text { развитая научно-образовательная инфраструктура. В случае длительного нахождения кла- } \\
\text { стера в данном состоянии можно определить его как лидера отрасли }\end{array}$ \\
\hline $0,9 \leq Q_{\text {эт }} \leq 1$ & $\begin{array}{l}\text { Эталонное развитие кластера. Считается, что предприятия-участники кластера испытыва- } \\
\text { ют небывалый рост производства и основная задача организации, управляющей кластером, } \\
\text { - предпринимать меры для удержания такого уровня производства в течение длительного } \\
\text { периода }\end{array}$ \\
\hline
\end{tabular}

Примечание. Составлено автором. 


\section{Заключение}

Развитие кластерных форм ведения экономической деятельности является одним из важнейших компонентов стратегической региональной политики. На данный момент существуют проблемы не только с описанием кластеров и их классификацией, но и с их идентификацией.

В многообразии информационного отражения кластеров как экономических систем разных уровней и назначений, масштабов и конфигураций, типов и сфер, родов и видов деятельности возможно применение многих моделей - математических, графических, логических и др. Их согласованное применение дает возможность определять стратегические и тактические меры развития кластеров, оценивать их место и роль в локальной, региональной, национальной и глобальной экономике.

Существующий инструментарий описания кластеров при помощи отдельных характеристик, предлагаемый отечественными методиками, не позволяет однозначно определить позиции кластера на локальном и глобальном рынках, особенности его функционирования и правильность принимаемых решений. Предложенные в работе подходы к информационному отражению кластера и расчету его обобщающего показателя позволяют приблизиться к решению вышеуказанных проблем.

На основании обобщающего показателя информационного отражения кластера могут приниматься более обоснованные стратегические решения разных уровней, направленные на повышение глобальной конкурентоспособности региона: влияние на подготовку высококвалифицированных специалистов, привлечение финансирования, совершенствование регуляторной политики, развитие транспортной инфраструктуры и логистики, развитие отрасли и межотраслевого взаимодействия, развитие экосистемы кластера.

Предложенная методика информационного отражения кластера может способствовать созданию паспортов экономических кластеров. Технология паспортизации хозяйственных единиц и их сложных ассоциированных (интегрированных или кооперированных) систем пока недостаточно развита в современ- ной экономике. Однако на современном этапе составление паспортов различных экономических образований является одним из трендов в разработке социально-экономической политики. Примером тому может служить составление паспортов муниципальных образований региона. Нельзя не отметить важность составления паспорта кластера, определения его параметров и характеристик для принятия стратегических и тактических решений в отношении кластера.

\section{СПИСОК ЛИТЕРАТУРЫ}

Идентификация кластера с учетом сравнительных преимуществ региона на примере Южного федерального округа / М. А. Боровская [и др.] // Региональная экономика: теория и практика. -2015 . - № 35. - С. 2-16.

Инновационные кластеры - лидеры инвестиционной привлекательности мирового уровня : метод. материалы / Е. А. Исланкина [и др.] ; Минэкономразвития России, АО «РВК», Нац. исслед. ун-т «Высшая школа экономики». - М. : НИУ ВШЭ, 2017. - 132 с.

Иншаков, О. В. Кластерное развитие российской наноиндустрии как стратегический компонент «умной специализации» регионов / О. В. Иншаков, Е. И. Иншакова // Региональная экономика. Юг России. - 2017. - №4(18). - С. 4-15. DOI: https://doi.org/10.15688/re.volsu.2017.4.1.

Марков, Л. С. Теоретико-методологические основы кластерного подхода / Л. С. Марков. - Новосибирск : ИЭОПП СО РАН, 2015. - 300 с.

Миролюбова, Т. В. Закономерности и факторы формирования и развития региональных кластеров : монография / Т. В. Миролюбова, Т. В. Карлина, Т. Ю. Ковалева ; Перм. гос. нац. иссл. ун-т. Пермь : [б. и.], 2013. -283 с.

Портер, М. Международная конкуренция. Конкурентные преимущества стран : пер. с англ. / М. Портер. - М. : Международные отношения, 1993. - $896 \mathrm{c.}$

Портер, М. Конкуренция. : пер. с англ. / М. Портер. М. : Вильямс, 2005.-608 с.

Реестр. Карта кластеров России // Российская кластерная обсерватория. 2019. - Электрон. текстовые дан. - Режим доступа: http://map. cluster.hse.ru/list. - Загл. с экрана.

Реестр промышленных кластеров // Геоинформационная система индустриальных парков, технопарков и кластеров Российской Федерации. 2019. - Электрон. текстовые дан. - Режим доступа: https://www.gisip.ru/reg_clusters. 
Clark, J. Tech Country. Looking Beyond London in Search of Britain's Technological Future. 2013. - Electronic text data. - Mode of access: https://www.academia.edu/3056231/Tech Country_Looking_beyond_London_in_search of_Britains_technological_future.-Title from screen.

\section{REFERENCES}

Borovskaya M.A., Shevchenko I.K., Razvadovskaya Y.V., Fedotova A.Yu., 2015. Identifikatsiya klastera s uchetom sravnitelnykh preimushchestv regiona na primere Yuzhnogo federalnogo okruga [Cluster Identification, Taking into Account the Comparative Advantages of the Region: The Southern Federal District Case Study]. Regionalnaya ekonomika: teoriya i praktika [Regional Economics: Theory and Practice], 2015, no. 35, pp. 2-16.

Islankina E.A., Kutsenko E.S., Rudnik P.B., Shadrin A.E. Innovatsionnye klastery-lidery investitsionnoy privlekatelnosti mirovogo urovnya: metod. materialy [Innovative Clusters - World-Class Leaders of Investment Attractiveness. Guidelines]. Moscow, NIU VShE, 2017. $132 \mathrm{p}$.

Inshakov O.V., Inshakova E.I. Klasternoe razvitie rossiyskoy nanoindustrii kak strategicheskiy komponent «umnoy spetsializatsii» regionov [Cluster Development of Russian Nanoindustry as a Strategic Component of Smart Specialization of the Regions]. Regionalnaya ekonomika. Yug Rossii [Regional Economy. South of Russia],
2017, no. 4 (18), pp. 4-15. DOI: https://doi.org/ 10.15688/re.volsu.2017.4.1.

Markov L.S. Teoretiko-metodologicheskie osnovy klasternogo podkhoda [Theoretical and Methodological Bases of Cluster Approach]. Novosibirsk, IEOPP SO RAN, 2015.300 p.

Mirolyubova T.V., Karlina T.V., Kovaleva T.Yu. Zakonomernosti $i$ faktory formirovaniya $i$ razvitiya regionalnykh klasterov [Tendencies and Factors of Creation and Development of Regional Clusters]. Perm, 2013. 283 p.

Porter M. Mezhdunarodnaya konkurentsiya. Konkurentnye preimushchestva stran [International Competition. Competitive Advantage of Nations]. Moscow, Mezhdunarodnye otnosheniya Publ., 1993.896 p.

Porter M. Konkurentsiya [On Competition]. Moscow, Vilyams Publ., 2005. 608 p.

Reestr. Karta klasterov Rossii [Registry. Map of Clusters of Russia]. Rossiyskaya klasternaya observatoriya [Russian Cluster Observatory], 2019. URL: http://map.cluster.hse.ru/list.

Reestr promyshlennykh klasterov [Register of Industrial Clusters]. Geoinformatsionnaya sistema industrialnykh parkov, tekhnoparkov i klasterov Rossiyskoy Federatsii [Geographic Information System of Industrial Parks, Science and Technology Parks and Clusters of the Russian Federation], 2019. URL: https:/www. gisip.ru/reg_clusters.

Clark J. Tech Country. Looking Beyond London in Search of Britain's Technological Future, 2013. URL: https:// www.academia.edu/3056231/Tech Country_Looking_beyond_London_in_search_of_ Britains_technological_future.

\section{Information About the Author}

Aleksandr E. Vorobev, Postgraduate Student, Department of Economic Theory, Regional and World Economics, Volgograd State University, Prosp. Universitetsky, 100, 400062 Volgograd, Russian Federation, vorobyov@volsu.ru, https://orcid.org/0000-0003-1771-6569

\section{Информация об авторе}

Александр Евгеньевич Воробьев, аспирант кафедры экономической теории, мировой и региональной экономики, Волгоградский государственный университет, просп. Университетский, 100, 400062 г. Волгоград, Российская Федерация, vorobyov@volsu.ru, https://orcid.org/0000-0003-1771-6569 